\title{
A Rare Case Report of Non-toxigenic Corynebacterium diphtheriae Bloodstream Infection in an Uncontrolled Diabetic With Peripheral Vascular Disease
}

\author{
Lakshmi Shanmugam ${ }^{1}$, Ketan Priyadarshi ${ }^{1}$, Mahalakshmi Kumaresan ${ }^{1}$, Monika Sivaradjy ${ }^{1}$, Praveen \\ Upadhyay $^{2}$, TP Elamurugan ${ }^{2}$, Apurba S. Sastry ${ }^{3}$ \\ 1. Microbiology, Jawaharlal Institute of Postgraduate Medical Education and Research, Puducherry, IND 2. Surgery, \\ Jawaharlal Institute of Postgraduate Medical Education and Research, Puducherry, IND 3. Microbiology: Hospital
} Infection Control, Jawaharlal Institute of Postgraduate Medical Education and Research, Puducherry, IND

Corresponding author: Apurba S. Sastry, drapurbasastry@gmail.com

Review began 04/27/2021 Review ended 04/30/2021 Published 05/10/2021

\section{๑) Copyright 2021}

Shanmugam et al. This is an open access article distributed under the terms of the Creative Commons Attribution License CC-BY 4.0., which permits unrestricted use, distribution, and reproduction in any medium, provided the original author and source are credited.

\begin{abstract}
Corynebacterium diphtheriae usually causes respiratory diphtheria, which is considered as a disease of toxemia but never bacteremia. Over the last few decades, cutaneous diphtheria has been increasingly reported owing to the emergence of the non-toxigenic strain, which causes locally necrotic and ulcerative lesions. Bacteremia is very rare, but the existing evidence in the literature suggests that the organism can rarely cause invasive infections such as septicemia, endocarditis, and osteoarthritis. Here, we present a rare case of $C$. diphtheriae causing bloodstream infections in an elderly diabetic with peripheral vascular disease, which was diagnosed incidentally on routine blood culture owing to automated identification systems viz matrix-assisted laser desorption ionization-time of flight (MALDI-TOF) confirmed with conventional methods, and susceptibility was performed using automated VITEK 2 system (BioMérieux, Marcy-l'Étoile, France), which has aided in the timely management.
\end{abstract}

Categories: General Surgery, Other

Keywords: corynebacterium diphtheriae, non-toxigenic, peripheral vascular disease, uncontrolled diabetes, gravis biotype, vitek 2, maldi tof ms, antimicrobial susceptibility

\section{Introduction}

Corynebacterium diphtheriae is an aerobic, gram-positive, non-motile, non-spore-forming, non-capsulated, non-fastidious, club-shaped gram-positive bacillus. The most common clinical presentation of this organism is respiratory diphtheria, a toxin-mediated disease characterized by the formation of pseudomembrane in various respiratory sites. However, over the last few decades, cutaneous diphtheria has been increasingly reported owing to the emergence of the non-toxigenic strain, subsequent to widespread vaccination programs [1].

Traditionally diphtheria is considered a disease of toxemia but never bacteremia. Bacilli are noninvasive, are present only at the localized site (pharynx), and secrete the toxin that spreads via bloodstream to various organs. Cutaneous diphtheria is also locally necrotic and ulcerative. But the existing evidence in the literature suggests that the organism can rarely cause invasive infections such as septicemia, endocarditis, and osteoarthritis [1]. Here, we present a rare case of $C$. diphtheriae causing bloodstream infections in a diabetic individual with peripheral vascular disease.

\section{Case Presentation}

A 63-year-old non-hypertensive diabetic male, farmer by profession, presented with complaints of blackish discoloration of distal part of the left foot for 15 days predominantly involving four small toes. He had similar blackish discoloration of left great toe for last one month, which was amputated 15 days back in a local hospital. He also complained of ulcerative wound over the distal part of left foot and amputated great toe (Figure 1). He was diagnosed as a case of uncontrolled type-2 diabetes mellitus with poor glycemic control on irregular oral hypoglycemics for five years. He had a history of claudication pain in lower limbs for the last one year, which gradually progresses to pain even at rest and loss of sensation of digits for the last three months. He is a chronic smoker and alcoholic for the past 20 years. There was no history of fever, pus discharge from the distal lower limb, chest pain, blurring of vision, blackouts, or abdominal pain. 


\section{Cureus}

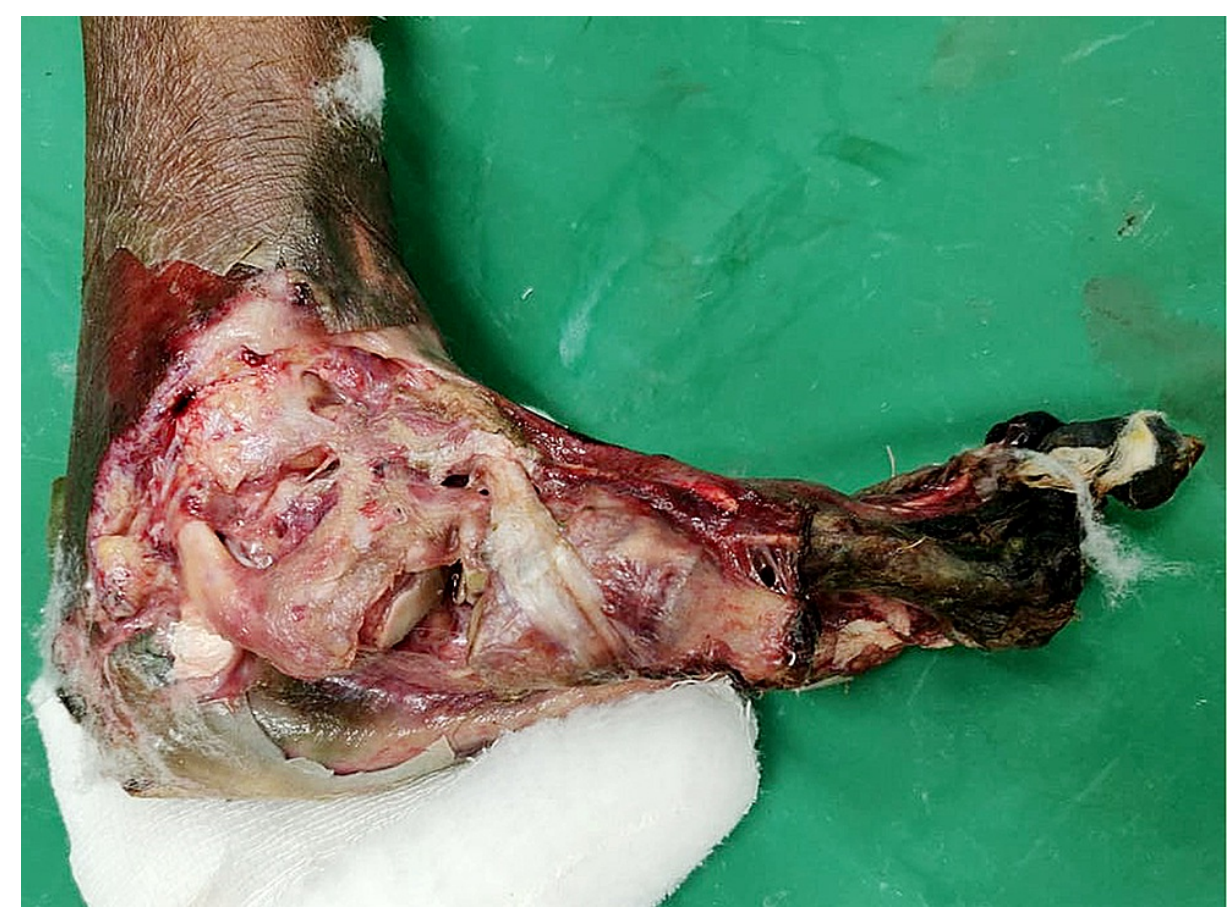

FIGURE 1: Post debridement status of gangrenous necrotic ulcer over the distal region of left foot

On clinical examination, there were no signs of pallor, icterus, pedal edema, lymphadenopathy, clubbing, or cyanosis with random blood glucose of $344 \mathrm{mg} / \mathrm{dl}$. Extensive ulceration and necrosis were present over the ankle, heel, and great toe region of left lower limb along with blackish discoloration of second to fifth toes. The peripheral arterial pulses were not palpable in all major peripheral arteries of both lower limbs. Computed tomograghy (CT) angiogram of lower limbs showed high-grade occlusion and luminal narrowing in almost all major arteries of both lower limbs. The provisional diagnosis was made as bilateral lower limb peripheral vascular disease due to uncontrolled diabetes mellitus. The patient was planned for above-knee amputation of left lower limb.

The patient had developed few episodes of mild grade fever, for which automated blood culture was collected and sent to the microbiology laboratory. The bottle was loaded in BacT/Alert Virtuo system (BioMérieux, Marcy-l'Étoile, France), which flagged positive signal after 26 hours of aerobic incubation at $37^{\circ} \mathrm{C}$. Gram staining was performed directly from flagged broth, which showed short gram-positive bacilli arranged in pairs with acute angular fashion (cuneiform arrangement) as shown in Figure 2. 


\section{Cureus}

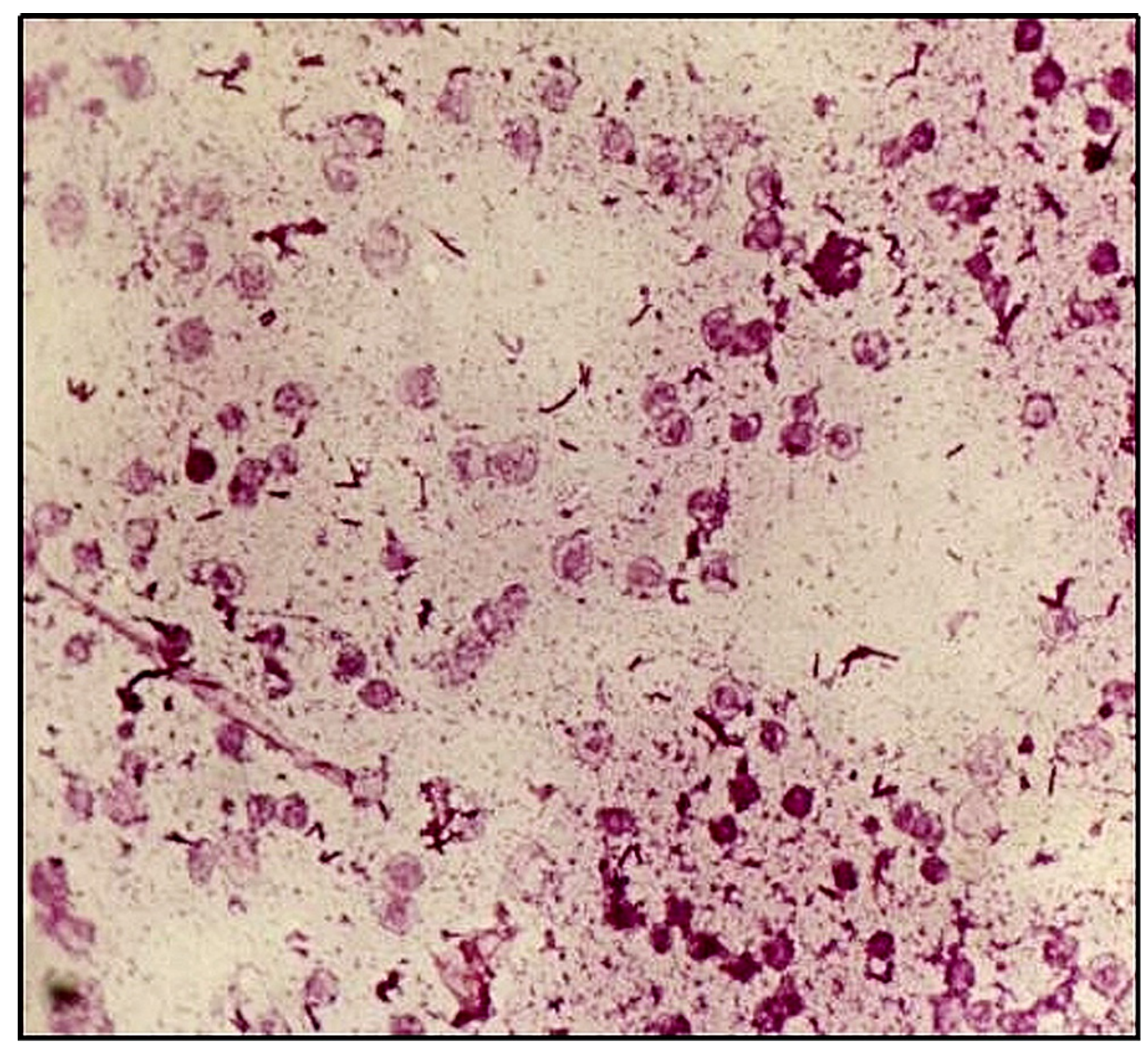

FIGURE 2: Gram stain from blood culture broth showing club-shaped gram-positive bacilli in cuneiform arrangement

Subculture from bottle was performed on $5 \%$ sheep blood agar (SBA), chocolate agar (CA), and MacConkey agar (MAC) and incubated aerobically at $37^{\circ} \mathrm{C}$. After 10 to 12 hours of incubation, minute grayish circular low-convex hemolytic colonies were seen on SBA (Figure 3). Gram staining from the colonies grown on SBA showed short gram-positive bacilli in cuneiform arrangement with swelling on one or both ends (club-

shaped). This is followed by Albert staining from the colony, which showed green-colored bacilli with bluishpurple metachromatic granules in cuneiform arrangement. Identification of the organism from the colony was obtained using matrix-assisted laser desorption ionization-time of flight mass spectrometry (MALDITOF MS) (VITEK MS version 3.0). It was identified as C. diphtheriae with a confidence interval of $99 \%$. Later, the identification was also confirmed by conventional method. 


\section{Cureus}

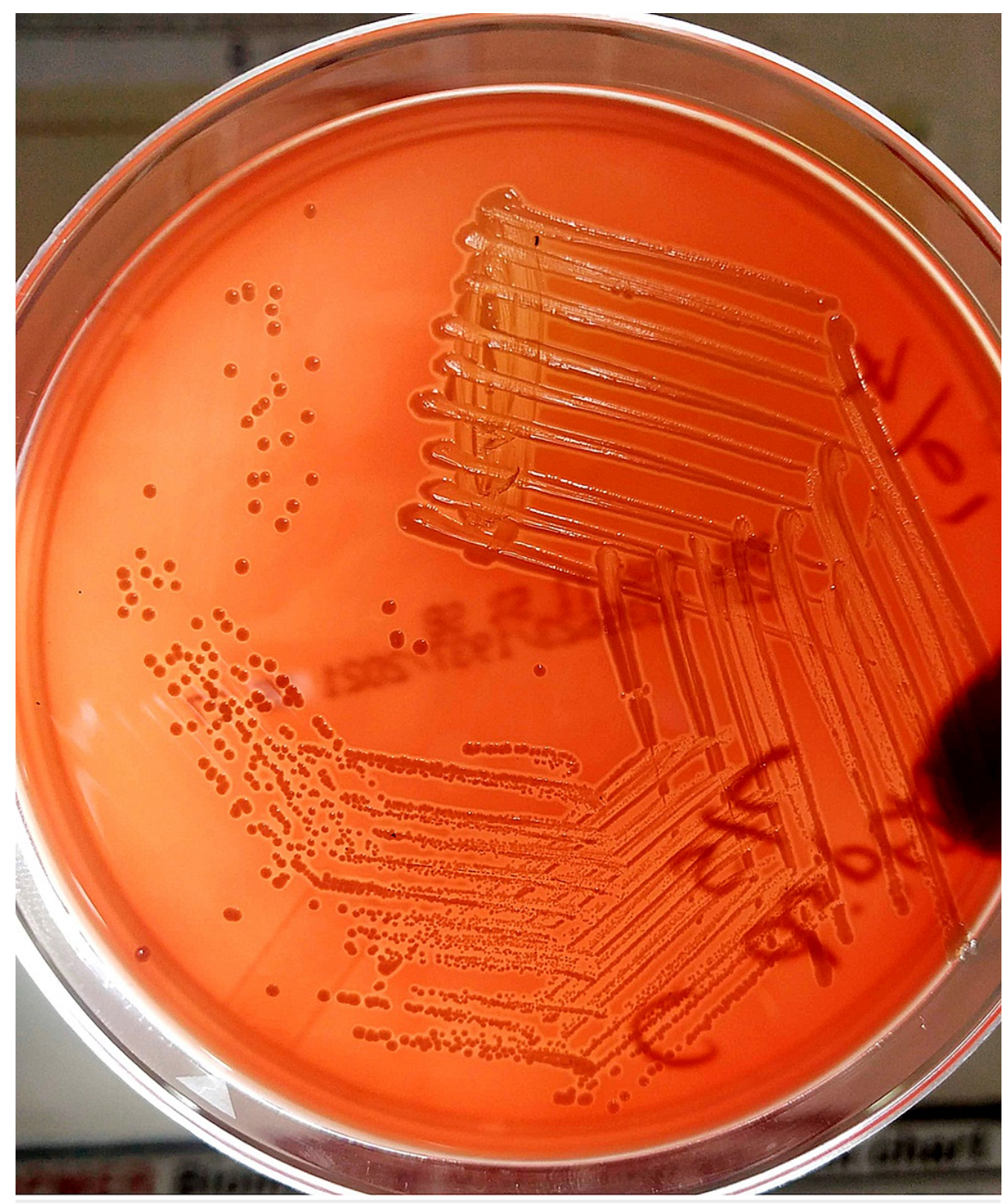

\section{FIGURE 3: Growth on $5 \%$ sheep blood agar showing minute grayish}

circular low-convex hemolytic colonies

Urea was not hydrolyzed; it produced black-colored colonies on potassium tellurite blood agar (KTBA) and fermented glucose, maltose, and starch with production of acid but no gas on Hiss's serum sugar fermentation media (Figure 4). The isolate was identified as "gravis" biotype. Antimicrobial susceptibility testing (AST) was performed by broth microdilution method using VITEK-2-automated AST system (ASTP628 card for gram-positive cocci). The result was interpreted using clinical breakpoints given in Clinical and Laboratory Standards Institute (CLSI) M-45 document [2]. The isolate was susceptible for benzylpenicillin, erythromycin, gentamicin, vancomycin, clindamycin, linezolid, and rifampicin, intermediate to ciprofloxacin and resistant to cotrimoxazole and tetracycline. In-house conventional PCR for tox gene (tox $A$ and tox $B$ ) and $d t x R$ gene was performed from the culture growth. The isolate was negative for tox genes but positive for $d t x R$ gene, which confirms that the isolate belongs to a non-toxigenic strain of C. diphtheriae, biotype gravis. 


\section{Cureus}

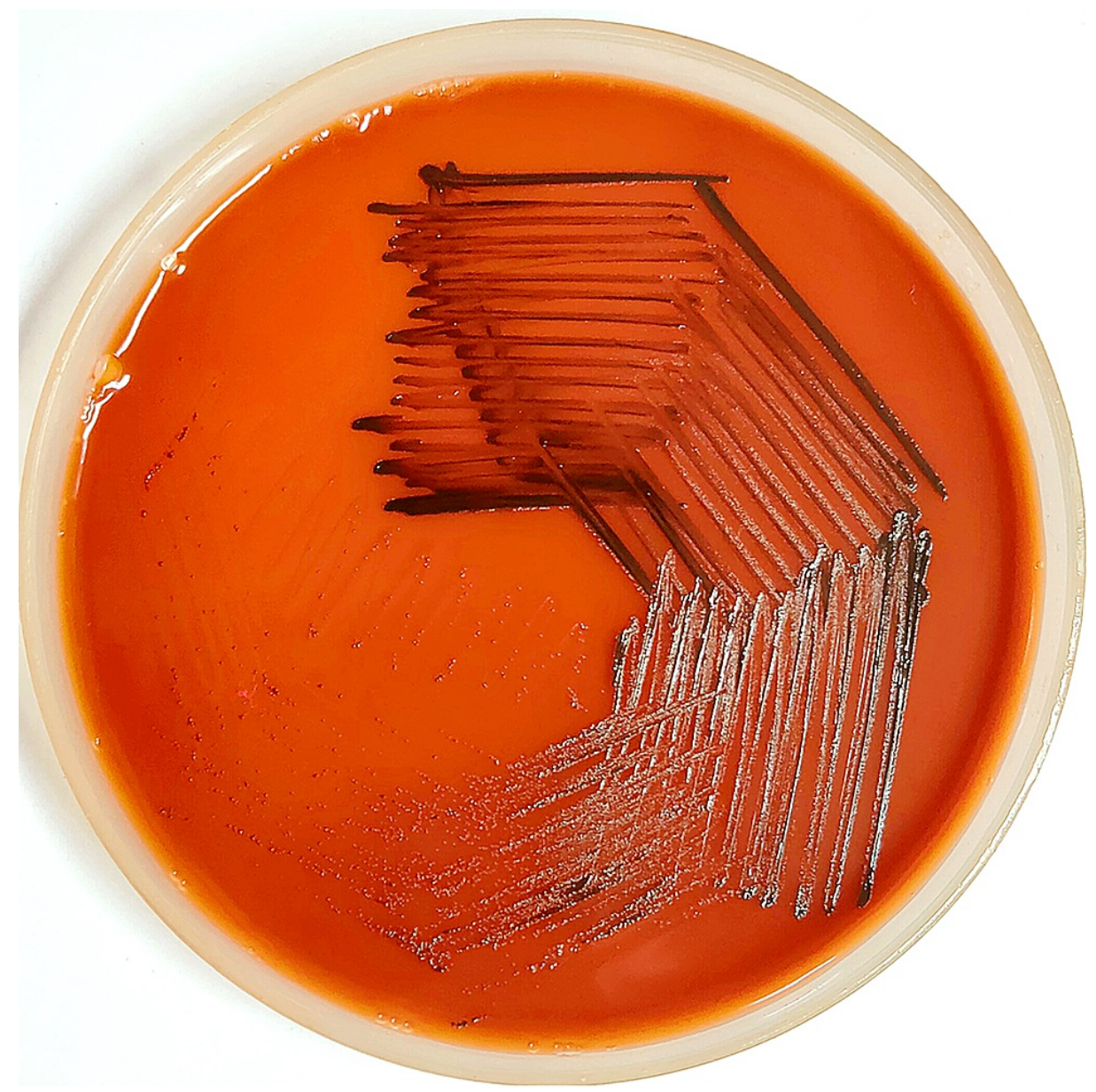

FIGURE 4: Growth on potassium tellurite blood agar showing jet black colonies

Following amputation, intra-operative necrotic tissue bits were sent for bacterial culture, which grew Providencia rettgeri and Enterococcus faecalis. The laboratory failed to show any growth of C. diphtheriae from tissue bit, possibly due to heavy gram-negative bacilli growth. Subsequently, a culture of throat swab specimen was also performed to find out any colonization, which failed to have any growth of $C$. diphtheriae. Therefore, the source of $C$. diphtheriae bacteremia was not properly established. The patient was empirically started on amikacin as pre-surgical prophylaxis since admission and subsequently changed to amoxycillinclavulanate and clindamycin based on the AST report. The patient improved clinically and was discharged on the same regimen.

\section{Discussion}

Diphtheria is generally a non-invasive disease characterized by toxemia but never bacteremia. However, the isolation of $C$. diphtheriae in blood, though rare, has always been regarded as significant in the existing literature.

There have been case reports of $C$. diphtheriae septicemia [1,3,4], infective endocarditis (native valve [5] and prosthetic valve [6]), and septic arthritis [7] due to non-toxigenic strains among varied age-groups viz child [1,4], adults [3], and risk-groups viz smokers [3], acute myeloid leukemia [1], congenital cardiopathy [4], etc. Only 58 cases of bacteremia infections were described between 1893 and 2003 due to this organism [8].

The most important virulence factor of C. diphtheriae is diphtheria toxin (DT). However, the invasive infections are mostly caused by non-toxigenic strains, which suggests that there could be other possible virulence factors such as adhesins, haemagglutinins, and surface-exposed non-fimbrial proteins. Extensive diphtheria vaccination programs (78\% DPT vaccine coverage rate in India [8]) that aim at reducing toxinmediated diseases has created a selection pressure resulting in the acquisition of other virulence factors like transposons, plasmids, etc. that might contain isolated virulence genes or pathogenicity island as a whole [3]. There are in-vitro studies, which suggest that invasiveness is because of a zipper-like mechanism mediated by some cell surface receptors, the details of which remain unclear [9]. 
In past, identification of $C$. diphtheriae was solely based on conventional methods such as morphological appearance, Albert stain findings, growth on KTBA, and biochemical properties are now strengthened by automated identification methods like MALDI-TOF MS [3]. Here, we have used a combination of the conventional method and MALDI-TOF MS for the identification and biotyping of $C$. diphtheriae. Available literature says that the accuracy of the MALDI-TOF MS system for the identification of $C$. diphtheriae, $C$. pseudotuberculosis, and C. ulcerans is very high (97\%-100\%) [9]. Toxin detection can be done by Elek's gel precipitation test [1,3] or PCR [3] for detection of genes such as tox, $d t x R$, 16S ribosomal RNA (16S rRNA), RNA polymerase $\beta$ subunit ( $r p o B$ ), etc. We have performed conventional PCR for tox and gene. We have determined minimum inhibitory concentration (MIC) by VITEK-2-automated AST system and interpreted using CLSI MIC breakpoints, similar to Wojewoda et al. who have determined MIC using microbroth dilution method $[1,3]$.

This is a rare case of non-toxigenic $C$. diphtheriae bloodstream infection (BSI) in a diabetic individual with peripheral vascular disease. In the present case, the patient was having risk factors like diabetes mellitus and peripheral vascular disease with gangrenous lower limb ulcers. The postulated hypotheses are as follows: (i) organisms gain access from the environment into the poorly vascularized gangrenous limb with breached skin barrier, multiply in the site, and cause extensive necrosis of the distal lower limb and subsequent entry into the bloodstream. (ii) BSI can be preceded with asymptomatic colonization of the upper respiratory tract in an apparently immunocompromised host. But in this study, we failed to attribute the source of BSI to either of these, as the culture of both throat swab and tissue bit from the wound did not yield C. diphtheriae. The possible reasons may be (i) prior institution of susceptible antimicrobial therapy viz amikacin before sending culture samples or (ii) the wound tissue had a heavy growth of a mixture of gram-negative organisms on SBA and MAC, because of which C. diphtheriae could not have been isolated. (iii) Repeat sampling was not possible because the patient underwent amputation.

\section{Conclusions}

This case highlights an unusual presentation of non-toxigenic C. diphtheriae bacteremia in a patient with immunocompromised comorbidities. This emphasizes sending appropriate clinical samples for microbiological cultures including blood culture before the institution of empirical antimicrobial therapy. The present case is a laboratory-guided incidental finding of $C$. diphtheriae BSI, most possibly preceded by cutaneous diphtheria. Automated identification and AST system help in arriving at an accurate and timely diagnosis for the appropriate management of the patient.

\section{Additional Information}

\section{Disclosures}

Human subjects: Consent was obtained or waived by all participants in this study. Conflicts of interest: In compliance with the ICMJE uniform disclosure form, all authors declare the following: Payment/services info: All authors have declared that no financial support was received from any organization for the submitted work. Financial relationships: All authors have declared that they have no financial relationships at present or within the previous three years with any organizations that might have an interest in the submitted work. Other relationships: All authors have declared that there are no other relationships or activities that could appear to have influenced the submitted work.

\section{Acknowledgements}

We would like to extend our heartful thanks to Dr. Sujatha Sistla, professor, faculty in charge of exudate and respiratory, ENT, and ophthal specimens section, JIPMER, Puducherry, India, for guiding us. We would also like to acknowledge Dr. Soundarya, Dr. Ramit Kundu, and Junior residents of exudate section for helping us by providing details from the exudate section.

\section{References}

1. Wojewoda CM, Koval CE, Wilson DA, Chakos MH, Harrington SM: Bloodstream infection caused by nontoxigenic Corynebacterium diphtheriae in an immunocompromised host in the United States. J Clin Microbiol. 2012, 50:2170-2. 10.1128/JCM.00237-12

2. Hindler JA, Richter SS, Bernard K, et al.: Methods for Antimicrobial Dilution and Disk Susceptibility Testing of Infrequently Isolated or Fastidious Bacteria. Clinical and Laboratory Standards Institute, 2015.

3. Zasada AA, Zaleska M, Podlasin RB, Seferynska I: The first case of septicemia due to nontoxigenic Corynebacterium diphtheriae in Poland: case report. Ann Clin Microbiol Antimicrob. 2005, 4:8. 10.1186/1476-0711-4-8

4. Berardi-Grassias L, Palette X, Richardin F, Guilhem C: [Corynebacterium diphtheriae septicemia in a child with congenital cardiopathy]. Presse Med. 1992, 21:1822-3.

5. Pachirat O, Kaewkes D, Pussadhamma B, Watt G: Corynebacterium diphtheriae native aortic valve endocarditis in a patient with prosthetic mitral valve: a rare presentation. Cardiol Res. 2018, 9:314-7. $10.14740 / \mathrm{cr} 741 \mathrm{w}$

6. Muttaiyah S, Best EJ, Freeman JT, Taylor SL, Morris AJ, Roberts SA: Corynebacterium diphtheriae endocarditis: a case series and review of the treatment approach. Int J Infect Dis. 2011, 15:e584-8. 10.1016/j.ijid.2011.04.003 


\section{Cureus}

7. Damade R, Pouchot J, Delacroix I, Boussougant Y, Vinceneux P: Septic arthritis due to Corynebacterium diphtheriae. Clin Infect Dis. 1993, 16:446-7. 10.1093/clind/16.3.446

8. Why is the Vaccination rate low in India? . (2021). Accessed: March 4, 2021:

https://arxiv.org/ftp/arxiv/papers/2103/2103.02909.pdf.

9. Sharma NC, Efstratiou A, Mokrousov I, Mutreja A, Das B, Ramamurthy T: Diphtheria. Nat Rev Dis Primers. 2019, 5:81. 10.1038/s41572-019-0131-y 